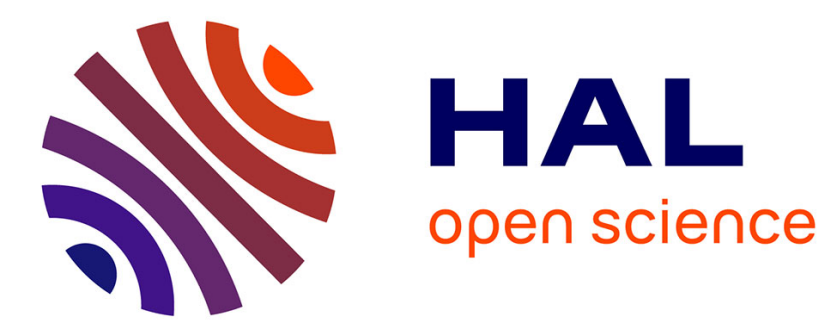

\title{
La pompe à pistons dans l'Antiquité
}

Philippe Fleury

\section{To cite this version:}

Philippe Fleury. La pompe à pistons dans l'Antiquité. Aquam in altum exprimere. Les machines élévatrices d'eau dans l'Antiquité , Mar 2003, Bordeaux, France. pp.139-151, 10.4000/books.ausonius.10479 . hal-01718780

\section{HAL Id: hal-01718780 \\ https://hal.science/hal-01718780}

Submitted on 19 Jul 2018

HAL is a multi-disciplinary open access archive for the deposit and dissemination of scientific research documents, whether they are published or not. The documents may come from teaching and research institutions in France or abroad, or from public or private research centers.
L'archive ouverte pluridisciplinaire HAL, est destinée au dépôt et à la diffusion de documents scientifiques de niveau recherche, publiés ou non, émanant des établissements d'enseignement et de recherche français ou étrangers, des laboratoires publics ou privés. 


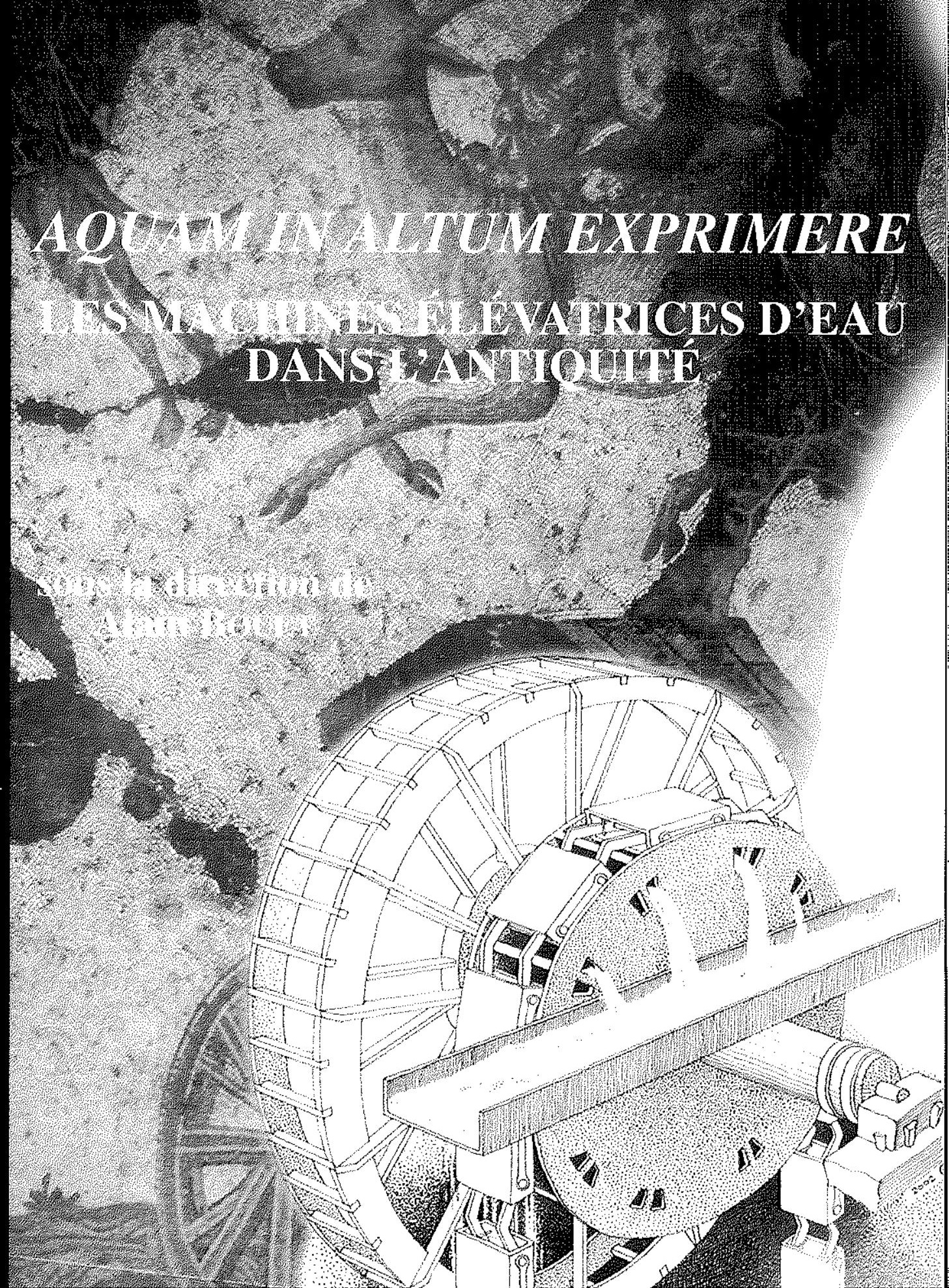




\section{SOMMAIRE}

AVANT-PROPOS

Alain BOUET, Le point de la recherche sur les machines élévatrices d'eau

Sophie COADIC, Alain BouET, La chaîne à godets des thermes de

Barzan (Charente-maritime) : une première approche

Francis FOURNIER, Philippe JACQUES, Le puits antique des Augustins à Layrac (Lot-et-garonne). Présence d'une roue

AUSONIUS

Maison de l'Archéologie
Université Michel de Montaigne - Bordeaux 3

Éric Plassot, Christophe Pellecuer, Puiser, élever et distribuer l'eau dans la villa de Careiron et Pesquier

à Milhaud (Gard) : premiers commentaires

F - 33607 Pessac Cedex

http://www-ausonius u-bordeaux 3.fr/EditionsAusonius

\section{CSs}

DIFFUSION DE BOCCARD

11 rue de Médicis

75006 Paris

http://www.deboccard.com

Directeur des Publications : Jérôme FRANCE Secrétairc des Publications : Nathalie TRAN Graphisme de couverture : Stéphanic VINCENT (C) AUSONIUS 2005

ISSN : $1298-1990$

ISBN : 2-910023-57-5

Achevé d'imprimer sur les presses

de l'imprimerie La Nef-Chastrusse

87 , quai de Brazza - BP 28

F - 33015 Bordeaux cedex

janvier 2005

Ian BLAIR, Robert SPAIN, Tony TAYLOR, The technology of the 1st - and 2nd century roman bucket chains from London : from excavation to reconstruction.

Margherita BEDELlo TATA, Stefania FogAGnolo, Una ruota idraulica da Ostia

Philippe FleUry, La pompe à pistons dans l'Antiquité

Éric Morvillez, Pascale CheVAliER, Jagoda MARDEšÍ́, Branko PENĐER, Mira ToPIĆ, Morana ČAUŠEviĆ, La noria découverte proximité de "l'oratoire A", dans le quartier épiscopal de Salone (mission archéologique franco-croate de Salone) ......... 
La pompe à eau à pistons naît probablement à Alexandrie, dans la première moitié du III $\mathrm{s}$. a.C., dans le contexte des travaux de Ctésibios sur la pompe à air pour l'orgue hydraulique et sur la catapulte à air comprimé. L'ouvrage de Philon de Byzance intitulé Pneumatiques, au milieu du III s. a.C., est le premier texte conservé à mentionner (et à décrire) la pompe à eau. Ensuite nous avons un "trou" de deux siècles, jusqu'au texte du $D e$ architectura de Vitruve qui décrit la "machine de Ctésibios" (machina Ctesibica) vers 25 a.C. Entre deux, aucune mention dans les textes, aucun témoignage archéologique; les plus anciens restes de pompe découverts datent du début du Irr s. p.C.

Nous laisserons de côté le texte de Philon qui pose divers problèmes de transmission pour commencer notre étude au Ier s. a.C. qui nous paraît marquer le début de la véritable diffusion de cet appareil. L'essentiel de notre enquête porte donc sur la période impériale romaine avec juste une "excursion tardive" dans les textes des lexicographes et encyclopédistes du $\mathrm{VI}^{\mathrm{e}}-\mathrm{VII}{ }^{\mathrm{e}} \mathrm{s}$. p.C. Nous parcourrons d'abord les sources littéraires et épigraphiques, puis les sources archéologiques avant de nous arrêter sur les textes de Vitruve et de Héron d'Alexandrie qui nous permettront d'approfondir les modes de construction et de fonctionnement de la pompe à pistons destinée à puiser de l'eau.

\section{TÉMOIGNAGES LITTÉRAIRES ET ÉPIGRAPHIQUES}

Dans la littérature latine, la pompe à pistons n'est décrite que par Vitruve ${ }^{1}$ au ${ }^{\text {er }} \mathrm{s}$. a.C., mais de façon suffisamment précise pour que nous puissions la reconstituer au moins dans ses grands principes (cf. infra). Dans

* CERLAM, Université de Caen, Basse-Normandie.

1 Vitr. 10.7.1-3. 
la littérature grecque, elle est décrite par Philon de Byzance ${ }^{2}$ au III ${ }^{\mathrm{e}}$ s. a.C., et par Héron d'Alexandrie ${ }^{3}$ au $\mathrm{r}^{e r}$ s. p.C. La description d'Héron est également suffisamment précise pour que nous puissions reconstituer sa machine, légèrement différente de celle de Vitruve (cf. infra). Son texte est même plus complet que celui de l'auteur latin (deux fois plus long en fait) et il nous donne davantage de détails sur les clapets, sur les leviers de manœuvre, et surtout sur le tuyau de sortie, complètement omis par Vitruve.

Hormis ces textes, nous ne trouvons dans la littérature que des mentions éparses qui nous permettent cependant d'appréhender les domaines d'utilisation. Ces mentions ne sont pas toujours faciles à repérer pour des questions de vocabulaire. Le terme spécialisé pour désigner la pompe à pistons est en latin sipho (on trouve également les formes sipo et sifon) et en grec oí $\omega v$, mais l'appareil peut aussi être mentionné avec des mots plus génériques. Vitruve par exemple n'utilise jamais le mot sipho. Pour sa description du livre X, il emploie l'expression machina ctesibica, "la machine de Ctésibios", en référence à son inventeur présumé.

En latin Vitruve constitue, nous l'avons dit, notre première mention de la pompe à pistons. Les références de Forcellini ${ }^{4}$ et d'Ernout-Meillet ${ }^{5}$ à un soi-disant emploi de sipho chez Lucilius, cité par Cicéron ${ }^{6}$ reposent sur une mauvaise interprétation du texte donné par les manuscrits ${ }^{7}$. Après Vitruve, les mentions sont curieusement concentrées au $\mathrm{I}^{\mathrm{er}}$ s. p.C. Dans quatre cas, la pompe à pistons est utilisée comme élément de comparaison. Dans le poème anonyme de l'Aetna, il s'agit des effets des vents enfermés dans les canaux souterrains : "Finalement, poussé de l'endroit où il s'était concentré comme par des pompes à pistons (ueluti siponibus actus), le vent sort plein de furie et 1'Etna tout entier le crache avec des flammes" 8 . Chez Sénèque il s'agit de faire comprendre l'origine de la foudre: "La foudre est un feu ramassé qui est lancé de manière à être offensif. Il nous arrive de prendre de l'eau dans nos deux mains réunies et serrant les deux paumes l'une contre

2 Phil. Byz., Pneum., 64 et Pneum. append., 1 chap. 2.

3 Héron, Pneum., 1.28

4 Forcellini 1864

5 Ernout \& Meillet 1967

6 Cic., Fin., 2.8/23.

Les manuscrits portent des formes incompréhensibles: hrysizon, hirzison,

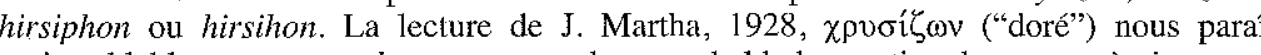
vraisemblable; en tout cas le contexte rend peu probable la mention de pompes à pistons.

8 Aetna, 327. l'autre, de la faire jaillir à la manière d'une pompe (in modum siponis)" 9 Pline l'Ancien utilise la pompe à pistons dans deux comparaisons; à propos de sources (ou de geysers?) : "L'eau irrigue la terre tout entière à l'intérieur comme à l'extérieur, au-dessus comme au-dessous, par les veines qui la parcourent comme autant de liens, et fait irruption même sur les sommets, où poussée par le souffle vital et exprimée par le poids de la terre elle jaillit à la manière des pompes (siphonum modo)" ${ }^{10}$; et à propos des sangsues: "Quand elles ont commencé à sucer, on les coupe avec des ciseaux et le sang s'écoule alors comme par des pompes (uelut siphonibus)" 11 .

Dans quatre autres cas la pompe à pistons est mentionnée pour ellemême. Deux fois dans le contexte des théâtres et des amphithéâtres pour la vaporisation d'eau parfumée, chez Sénèque le Rhéteur ${ }^{12}$ (siphonibus) et chez son fils, Sénèque le Philosophe. Dans ce dernier exemple l'allusion est indirecte, mais il ne fait guère de doute que le tormentum dont parle Sénèque est une pompe à pistons : "Tu ne doutes pas, je pense, que les jets de liquide qui, au milieu de l'arène, se développent des fondations et arrivent jusqu'aux gradins les plus élevés de l'amphithéâtre, ne soient un effet de la tension de l'eau? Eh bien ce n'est pas la main de l'homme, ni la machine (tormentum), mais l'air qui projette l'eau et la met en mouvement" 13 . Une fois dans un contexte d'irrigation, chez Pline l'Ancien, pour puiser l'eau des puits 14 (organis pneumaticis) et une fois pour lutter contre les incendies chez Pline le Jeune: "Il n'y a (à Nicomédée) aucune pompe (sipo) publique, aucun seau, enfin aucun matériel pour combattre les incendies" 15 . A ces exemples

9 Sen., Nat., 2.16 (Oltramare 1929)

10 Plin., Nat., 2.166, traduction Beaujeu 1950, sauf pour le mot siphonum que J. Beaujeu traduit par "siphon" qui nous parait ambigu.

Plin., Nat., 32.124 ; nous ne suivons pas l'interprétation d'E. de Saint-Denis 1966 qui traduit siphonibus par "tuyaux", car il nous semble que l'idée de "pression" est importante dans la comparaison de Pline.

${ }^{12}$ Sen., Contr., 10, pr. 9 : Quis enim ferat hominem de siphonibus dicentem "'aelo repluunt" et de sparsionibus "odoratos imbres".

${ }^{13}$ Sen., Nat., 2.16; du point de vue du fonctionnement, Sénèque commet ici la même erreur que Vitruve en pensant que c'est l'air qui pousse l'eau alors qu'en réalité la pression est exercée directement par les pistons (cf. infra).

${ }^{14}$ Plin., Nat., 19.60 : Hortos uillae iungendos non est dubium riguosque maxime habendos, si contingat, praefluo amne, si minuo, e puteo rota organisue pneumaticis uel tollenonum haustu rigatos. 
nous pourrions peut-être ajouter un passage de Columelle où il est question d'injecter des sirops sucrés dans les ruches des abeilles avec des siponibus ${ }^{16}$ qui pourraient ici être de petites pompes à pistons du type de celle de Bolsena II (cf infra), mais il peut aussi s'agir de simples tuyaux, sens assez répandu pour le mot sip $(h) o$.

Après le $\mathrm{I}^{\mathrm{er}} \mathrm{s}$. p.C. la pompe à pistons est mentionnée au début du II s. p.C. dans le Digeste ${ }^{17}$ (sifones) pour la lutte contre les incendies et chez Isidore de Séville au VII ${ }^{\mathrm{s}}$ s. p.C. qui la définit pour le même usage ${ }^{18}$.

Dans la littérature grecque correspondant à la période que nous avons définie, outre chez Héron d'Alexandrie, la pompe à pistons n'est mentionnée, à notre connaissance, que trois fois et toujours dans le contexte de la lutte contre les incendies: chez Apollodore de Damas au début du $\mathrm{II}^{\mathrm{e}} \mathrm{s}$. p.C. ( $\sigma$ íp $\mathrm{v}^{19}$ ) dans la Vie de Saint Polycarpe: au cours d'un incendie qui

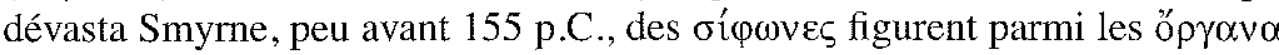
que fait apporter le stratège ${ }^{20}$, et chez Hésychius au $\mathrm{VI}^{\mathrm{e}} \mathrm{s}$. p.C. qui la défini comme un instrument pour projeter de l'eau sur les incendies. Il faut probablement ajouter à ces exemples un passage de Plutarque dans lequel la pompe elle-même n'est pas mentionnée, mais doit être utilisée pour asperger du parfum sur des convives: "Othon [...] avait [...] mis en œuvre des tuyaux $(\sigma \omega \lambda \hat{\eta} \vee \alpha \varsigma)$ d'or et d'argent qui lancèrent de tous les côtés à la fois des essences comme si c'eut été de l'eau et en inondèrent les convives" 21

Les inscriptions nous apportent en plus les mentions de "pompiers" sifonarii ${ }^{22}$

\section{TÉMOIGNAGES ARCHÉOLOGIQUES}

A ce jour dix-neuf pompes romaines (ou restes de pompes) ont été découvertes ; c'est un chiffre considérable (et providentiel) dans le domaine

${ }^{16}$ Colum. 9.14.17.

17 Dig. 337.12

18 Isid., Orig., 20.6.9: Sifon uas appellatum quod aquas sufflando fundat; utuntur enim hos oriente. Nam ubi senserint domum ardere, currunt cum sifonibus plenis aquis et extingunt incendia.

${ }^{19}$ Apollod., Poliorc., 7.7 (Thévenot 32d, Wescher 174, 5)

$460)$.

20. Pionus, Vita Sancti Polycarpi, 18 (éd. J. B. Lightfoot, Londres-New-York, 1889

${ }^{21}$ Plut., Gal., 19.3

${ }^{22}$ CIL, VI, 1057L 5, 1058R6, 2994, 3744 de la mécanique ancienne pour lequel l'archéologie est souvent d'un maigre secours. Elles datent toutes de l'époque impériale, aucune n'est antérieure au ${ }^{\text {er }}$ s. p.C. Nous ne ferons pas ici des descriptions détaillées que l'on retrouve aisément ailleurs ${ }^{23}$, mais après une énumération sommaire nous nous attacherons à montrer ce qu'apporte l'archéologie par rapport aux textes. Voici donc d'abord la liste des témoignages archéologiques.

- La Tradelière (France); début du I I ${ }^{\text {r }}$ s. p.C.; bronze; dans une épave.

- Dramont (France) ; milieu du Irer s. p.C. ; bronze ; dans une épave : ensemble avec quatre cylindres

- Saint-Malo (France) ; vers 100 p.C.; bois ; machinerie complexe dans un bassin.

- Trèves II (Allemagne); début du II ${ }^{\mathrm{e}}$ s. p.C.; bois ; dans l'amphithéâtre (pour assécher un passage souterrain?)

- Périgueux (France) ; avant la fin du II s. p.C. ; bois ; dans un puits.

- Zewen-Oberkirch (Allemagne) ; début du III' s. p.C. ; bois ; dans un puits.

- Wederath-Hunsrück (Allemagne) ; milieu du III ${ }^{\mathrm{e}}$ s. p.C. ; bois ; dans un puits.

- Bolsena I (Italie) ; avant la fin du III ${ }^{\mathrm{e}}$ s. p.C.? ; bronze ; contexte de la découverte incertain.

- Bolsena II (Italie) ; III s. p.C.? ; bronze ; dans une villa; modèle très petit : hauteur des cylindres $=11,5 \mathrm{~cm}$, diamètre intérieur $=$ $2,5 \mathrm{~cm}$.

- Silchester (Grande Bretagne) ; entre le II ${ }^{\mathrm{e}}$ s. et le $\mathrm{V}^{\mathrm{e}}$ s. p.C. ; bois ; dans un puits?

- Benfeld (France); époque impériale; bois; contexte de la découverte incertain.

- Castrum Novum (Italie) ; époque impériale ; bronze ; contexte de la découverte incertain.

- Metz (France) ; époque impériale ; bois ; dans un puits.

- Sotiel Coronada (Espagne); époque impériale ; bronze ; dans une galerie de mine.

${ }^{23}$ L'ouvrage fondamental est Oleson 1984 ; il faut y ajouter, pour la pompe de Périgueux (que J. P. Oleson ne connaît pas), Sablayrolles 1988, 141-196; pour la pompe de l'épave de Dramont nous avons aussi l'excellente étude de G. Rouanet, 1974, mais il faut en exclure le chapitre qui commente les textes de Vitruve et de Héron p. 73 et qui est erroné. 
- Trèves I (Allemagne) ; époque impériale ; bois ; dans un puits.

— Trèves III (Allemagne) ; époque impériale ; bois ; dans un puits.

- Colle Mentuccia (Italie) ; époque incertaine ; bois ; dans un puits.

- Rome-Antiquario Comunale, aujourd'hui disparue; époque incertaine; bronze; contexte de la découverte inconnu (parfois identifiée avec la pompe de Castrum Novum).

Ces témoignages viennent utilement compléter notre connaissance de la pompe à pistons romaine au moins dans six domaines.

A ne lire que les textes de Vitruve et d'Héron, nous aurions pu penser que ces pompes n'étaient faites qu'en bronze. Vitruve: "Cette machine doit être en bronze" 24 ; Héron : "On prend deux cylindres de bronze" 25 . Or sur les dix-neufs pompes ou éléments de pompes conservés, sept seulement sont en bronze. Le bois est donc le matériau privilégié pour presque les deux-tiers des pompes retrouvées. On pourrait certes arguer que le bronze étant un matériau recyclable, certains appareils ont pu être fondus pour d'autres utilisations, mais on peut aussi souligner que le bronze a mieux résisté au temps et aux incendies. Les deux phénomènes s'équilibrent probablement et il faut admettre que le bois était un matériau très utilisé, certainement pour la facilité de façonnage et son coût moindre.

Les témoignages archéologiques nous apportent aussi beaucoup de variantes dans les modes de construction et de fonctionnement. Si le principe de base est naturellement toujours le même, seulement six pompes sur les dix-neuf correspondent aux descriptions de Vitruve et de Héron que nous verrons dans la troisième partie. Il s'agit des pompes de Périgueux, Bolsena I et II, Castrum Novum et Sotiel Coronada.

Les textes ne disent rien sur la façon d'obtenir l'étanchéité entre piston et cylindre. Le texte de Vitruve laisse entendre que l'ajustage doit être parfait et les pistons seulement huilés. Cette possibilité a été vérifiée sur la pompe de Dramont, où le jeu entre cylindres et pistons est de l'ordre de $5 / 10 \mathrm{de} \mathrm{mm}$ et où, lors des essais, une graisse épaisse (plutôt que l" "huile" mentionnée par Vitruve) a permis d'obtenir une étanchéité parfaite ${ }^{26}$. Aucune pompe en bronze retrouvée ne présente les traces d'un autre moyen d'obtenir l'étanchéité mais, sur certaines pompes en bois, ont été repérées des traces de joint (disques en cuir d'une épaisseur de $4 \mathrm{~mm}$ fixés à l'extrémité des pistons

24 Vitr. 10.7.1 : Ea sit ex aere

${ }^{25}$ Héron, Pneum., 1.28 .

26 Rouanet 1974 sur la pompe de Périgueux par exemple ${ }^{27}$, ou rondelles de cuir également sur la pompe de Zewen-Oberkirch)

$\mathrm{Ni}$ Vitruve, ni Héron, ni bien entendu les autres témoignages épars ne nous donnent une quelconque indication sur les dimensions de ces appareils. Ce qui a pu être mesuré sur les pompes retrouvées fait apparaître une large gamme de tailles: le diamètre intérieur des cylindres va de $2,5 \mathrm{~cm}$ pour Bolsena II à $15 \mathrm{~cm}$ pour Saint-Malo, leur hauteur de $10,5 \mathrm{~cm}$ pour Dramont à $82 \mathrm{~cm}$ pour Périgueux.

Ces dimensions ainsi que des essais qui ont pu parfois être effectués comme sur la pompe de Dramont nous renseignent sur le débit des appareils. Il ne peut s'agir que d'évaluation car, pour connaître le débit théorique exact, il faudrait qu'outre le diamètre intérieur du cylindre nous ayons aussi la mesure précise de la course du piston ${ }^{28}$. Or celle-ci dépend de trois éléments que nous ne possédons jamais au complet : la distance entre chaque cylindre pour les pompes géminées, la longueur des bielles et celle des pistons. Un essai effectué sur des cylindres de la pompe de Dramont nous donne quand même quelques indications utiles. Le cylindre en question a un diamètre intérieur de $4,4 \mathrm{~cm}$, la course possible du piston est de $12 \mathrm{~cm}$ (sa forme creuse et la présence d'une chambre sous le cylindre lui permet d'avoir une course un peu plus longue que la hauteur propre du cylindre). Sur vingt essais, il a fallu une moyenne de quarante révolutions du piston pour remplir un récipient de 7 litres. Au rythme d'une révolution par seconde ${ }^{29}$ cela donne un débit de $10,5 \mathrm{l} / \mathrm{mn}$. Le débit théorique est de $11 \mathrm{l} / \mathrm{mn}$ : la différence est due aux effets de frottement, au temps de fermeture des clapets et à leur étanchéité qui peut ne pas être parfaite. Comme cette pompe fonctionnait avec quatre cylindres, son débit estimé est de $42 \mathrm{l} / \mathrm{mn}$. Le débit de la pompe de Périgueux (diamètre des cylindres : $8,6 \mathrm{~cm}$ et $9,7 \mathrm{~cm}$, course estimée des pistons : $21 \mathrm{~cm}$ ) est évalué à $60 \mathrm{l} / \mathrm{mn}$ (pour un débit théorique de $110 \mathrm{l} / \mathrm{mn}$ ). En revanche le débit théorique de la petite pompe de Bolsena II n'est que de $3,25 \mathrm{l} / \mathrm{mn}$, ce qui ne doit pas donner plus de 3 l en réalité.

Enfin, si la majeure partie des pompes retrouvées (et dont on connaît l'origine) l'a été dans des puits, conformément à un type d'utilisation signalé

27 Sablayrolles 1988

${ }^{28}$ Le volume d'eau déplacé $\left(\mathrm{en}^{\mathrm{cm}}{ }^{3}\right)$ pour un seul trajet de piston est donné par la formule $\pi R^{2} \times L(R=$ rayon du piston en $\mathrm{cm} ; \mathrm{L}=$ course du piston en $\mathrm{cm})$. ${ }^{29}$ Lors des essais, l'opérateur effectuait en réalité les 40 révolutions en l'espace de 
par Pline, le témoignage des pompes de la Tradelière et de Dramont nous montre un usage non signalé dans les textes : l'épuisement de l'eau de la cale des navires. Par ailleurs le fait que les pompes aient été majoritairement retrouvées dans des puits ne signifie pas que c'était là leur utilisation principale, mais simplement que les puits sont un lieu privilégié de la conservation de ce type d'objet ...

\section{MONTAGE ET FONCTIONNEMENT}

Nous l'avons vu par les témoignages archéologiques, à partir d'un principe de base unique : succion et refoulement de l'eau par le mouvement d'un piston dans un cylindre (fig. 1), il y a une grande variété de montages et de modes de fonctionnement. Une étude détaillée de toutes les variantes attestée dépasserait de loin le cadre de cet article (un examen des différents types de clapets pourrait faire l'objet d'un article à lui tout seul...). Nous nous en tiendrons donc aux montages décrits par Vitruve et Héron, attestés par six des dix-neuf pompes retrouvées.

3.1. La pompe de Vitruve (fig. 2)

"Il convient maintenant de décrire la machine de Ctésibios, qui porte l'eau en hauteur. Cette machine doit être en bronze. A sa base, et à faible distance l'un de l'autre, se trouvent deux cylindres jumeaux (B), munis de tuyaux (I) qui, formant une fourche, leur sont adaptés symétriquement et qui

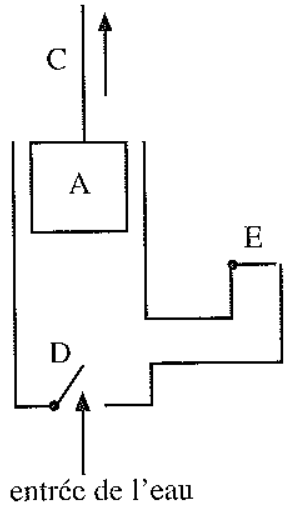

1. Sucion

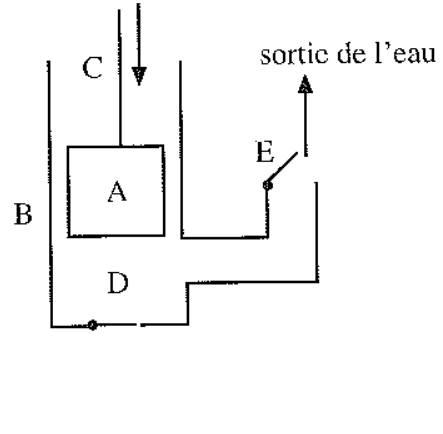

2. Refoulement

Fig. 1 : Principe de la pompe à piston.
A : piston

B : cylindre

C: biclle

D : clapct d'admission

E : clapet d'échappement

convergent sur un vase intermédiaire $(\mathrm{G})$. Dans ce vase se trouvent des clapets $(\mathrm{F})$, ajustés avec précision aux orifices supérieurs des tuyaux; obturant le passage de ces orifices, ils empêchent le retour de ce que l'air a chassé dans le vase. Au-dessus du vase est adaptée une chape $(\mathrm{J})$, en forme d'entonnoir renversé, qu'une clavette, passée dans une cheville, assujettit au vase afin d'éviter que la pression de l'eau ne la fasse se soulever. Un tuyau $(\mathrm{H})$, que l'on appelle trompe, est ajusté au-dessus, dressé verticalement. Sous les orifices inférieurs des tuyaux, les cylindres sont munis, par ailleurs, de clapets (E) appliqués sur les ouvertures qui sont dans leur fond. Cela étant, des pistons (C), emboîtés par le ${ }_{\mathrm{D}}$ :

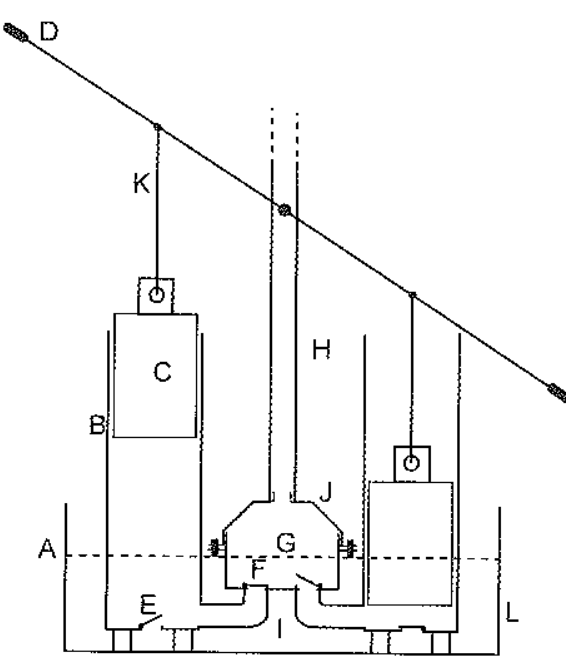

Fig. 2 : Pompe de Vitruve : niveau de l'eau $; \mathrm{B}:$ cylindre ; $\mathrm{C}$ : piston D : levier de manouvre ; E : clapet haut dans les cylindres, polis au tour et vase intermédiaire; $\mathrm{H}$ : tuyau de sortie ; frottés d'huile, sont entraînés par des vase ine sortie des cylindres; J : couvercle du bielles $(\mathrm{K})$ et des leviers (D) ; comme il y vase intermédiaire ; K : bielle; $\mathrm{L}:$ réservoir aura là de l'air avec l'eau, et les clapets d'eau.

obturant les ouvertures, ces pistons

pousseront et chasseront l'eau, par pression d'air, à travers les orifices des tuyaux, dans le vase ; de là elle sera recueillie dans la chape, d'où l'air la forcera à s'élever à travers le tuyau, jusqu'au haut; ainsi, depuis un endroit bas, et après installation d'un réservoir (L), l'eau peut-elle être amenée pour jaillir ${ }^{30 "}$.

Le texte de Vitruve est bref mais suffisant pour reconstruire l'appareil. Il contient cependant une mauvaise interprétation du rôle que jouerait l'air dans le montage. En admettant même que ce type de pompe ne fonctionne pas en immersion (ce qui est peu probable, cf. infra), l'air ne serait présent qu'au moment de l'amorçage. Une fois le système amorcé, il n'y a plus d'air dans le circuit, les pistons poussent et aspirent directement le liquide ${ }^{31}$.

${ }^{30}$ Vitr. 10.7.1-3 (Callebat \& Fleury 1986)

${ }^{31}$ Terquem $1885,85-86$, se trompe, à notre avis, en suivant Vitruve au pied de la 
L'erreur vient probablement d'une confusion avec l'orgue hydraulique (décrit par Vitruve au chapitre suivant) dans lequel les cylindres aspirent et refoulent effectivement de l'air, dont la compression est ensuite régulée par l'eau dans un compartiment intermédiaire. Nous interprétons le réservoir (castellum) mentionné à la fin de la description comme le bassin dans lequel est placée la pompe (qui fonctionne donc en immersion) et non comme un réservoir de collecte, interprétation de J. P. Oleson ${ }^{32}$. Le vase intermédiaire, absent dans le montage d'Héron, mais présent sur les pompes de Bolsena II et de Sotiel Coronada, nous paraît avoir un double intérêt. 1) Il permet d'utiliser des clapets d'admission et d'échappement de même type, fonctionnant tous en position horizontale, ce qui est le cas le plus favorable (Héron doit décrire deux types de clapets différents). 2) Il permet d'accéder facilement aux clapets d'échappement pour le nettoyage et le remplacement éventuel. Vitruve ne dit rien du système de projection de l'eau en aval du tuyau de sortie, mais cela est conforme à l'esprit général de son livre qui se veut une "encyclopédie condensée" : l'essentiel est pour lui d'expliquer les principes généraux de fonctionnement, non d'entrer dans les détails de fonctionnement.

\section{La pompe d'Héron (fig. 3)} suivante.

"Les pompes utilisées contre les incendies se construisent de la façon

Soient deux cylindres en bronze (B), dont la surface intérieure est travaillée au tour pour recevoir un piston, comme les cylindres des orgues hydrauliques, et les pistons correspondants (C). Les cylindres communiqueront entre eux au moyen du tube (I) et à l'intérieur du tube (I) les cylindres auront des clapets (F) ouvrant vers l'extérieur, comme ceux qui ont été décrits plus haut. Les cylindres auront aussi dans leur fond des trous circulaires obturés par des rondelles (E), à travers lesquelles passent des tiges soudées ou fixées de quelque autre manière au fond des cylindres, et ayant à leur extrémité un moyen pour empêcher les rondelles de-s'en aller. Les pistons auront, fixé en leur centre, une bielle (K), qu'on reliera à un balancier (D), mobile en son centre autour d'un axe fixe, et de part et d'autre les bielles (K) seront mobiles autour des axes $(\mathrm{O})$. Le tube $(\mathrm{I})$ communiquera avec un autre tube vertical $(\mathrm{H})$ qui se séparera en deux branches au point $(\mathrm{P})$, avec de petits tuyaux à travers lesquels on peut chasser l'eau, comme cela a déjà été dit plus haut dans la description du vase qui lance l'eau par la force de l'air comprimé. Si donc les cylindres munis de ces dispositions sont placés dans un vase plein d'eau (L), et si on imprime au balancier (D), à partir de ses extrémités un mouvement alternatif autour de l'axe, les pistons, en descendant, expulseront l'eau par le tube $(\mathrm{H})$ et par l'orifice mobile (M). En effet, un piston (C), en montant, ouvre le trou en faisant monter la rondelle (E) et il ferme le A Fig. 3 : Pompe d'Héron d'Alexandrie. clapet $(\mathrm{F})$; en descendant, il ferme levier de manouvre; $\mathrm{E}:$ : clapet d'admission ; $\mathrm{F}$ : (F), à d'échappement; $\mathrm{H}$ : tuyau de sortie ; I : tube l'eau est obligée de à travers lequel de sortie des cylindres; $\mathrm{K}$ : bielle ; $\mathrm{L}$ : réservoir menter levier; $P$ : jonction tuyau de sortie/orifice de sorti même résultat est obtenu aussi avec mobile.

l'autre piston. Le petit tuyau (M), qui peut tourner en avant et en arrière, permet de lancer l'eau à la hauteur voulue, mais non dans la direction voulue, à moins de déplacer la machine tout entière, ce qui entraîne des lenteurs et des difficultés en cas d'urgence. Pour que l'eau puisse être facilement lancée vers l'endroit voulu, on fera le tuyau $(\mathrm{H})$ en deux parties soigneusement ajustées l'une à l'autre dans le sens de la longueur, et l'une de ces parties sera fixée au tube (I), et l'autre au point de bifurcation (P) ; ainsi, par la rotation du tube supérieur et l'inclinaison de l'orifice $(M)$, le jet peut être lancé dans la direction que l'on veut. Le tuyau supérieur qui est embouti aura aussi des arrêts pour éviter qu'il soit séparé de la machine par la violence de l'eau. Ces arrêts seront en forme de $\Gamma$ et fixés au tube, et ils pourront glisser sur un anneau fixé au tube inférieur ${ }^{33}$ ".

Sur le fond, le système décrit par Héron diffère de celui de Vitruve essentiellement par l'absence de vase intermédiaire. Ce n'est pas un progrès, comme on l'écrit parfois, mais un montage différent. C'est surtout par la forme que les deux auteurs se distinguent l'un de l'autre. La description

\footnotetext{
33 Héron, Pneum., 1.28 (Argoud \& Guillaumin 1997).
} 
d'Héron est plus précise et plus longue. L'auteur grec utilise, pour chaque pièce de la machine, des sigles renvoyant à une figure aujourd'hui perdue ${ }^{34}$, il donne des détails sur le mode de fabrication des clapets, sur le système de balancier et sur le système de jet d'eau puisque son appareil est clairement orienté pour une utilisation dans la lutte contre les incendies.

\section{CONCLUSION}

La pompe à pistons est donc un objet mécanique sur lequel nous sommes exceptionnellement bien renseignés, à la fois par les textes et par les témoignages archéologiques. Seule l'iconographie fait défaut. Nous parlons d'"exception" car dans le domaine de la mécanique ancienne les sources textuelles sont rares (surtout pour la partie latine) et les témoignages archéologiques parfois inexistants. Son utilisation se répartit essentiellement dans quatre domaines. 1) La lutte contre les incendies : c'est l'utilisation la plus fréquemment mentionnée dans les textes et l'épigraphie. 2) La diffusion d'eau parfumée dans les théâtres et les amphithéâtres (nous pouvons y adjoindre la même utilisation, lors de banquets fastueux: l'exemple de Plutarque, à propos d'Othon, est là pour le montrer). La pompe de Trèves II avait peut-être cette utilisation. 3) L'élévation de l'eau proprement dite, en particulier du fond des puits: c'est l'utilisation la mieux attestée par l'archéologie. 4) Le pompage de l'eau dans les cales des navires. Pour ces deux derniers domaines, la pompe est en concurrence avec d'autres moyens mécaniques, notamment la chaîne à godets et la pompe à chapelets. Son intérêt essentiel est dans les deux premiers domaines et réside dans le fait de pouvoir envoyer un jet d'eau sous pression dans une direction précise : les emplois littéraires métaphoriques que nous avons relevés plus haut font tous référence à l'idée d'un jet d'eau sous pression. La pompe à pistons semble disparaître en même temps que l'empire romain. Elle réapparaît chez les ingénieurs de la Renaissance, dans les textes et les dessins de Mariano di Jacopo (dit Taccola) ${ }^{35}$ et de Paolo Santini, copie du De machinis de Taccola ${ }^{36}$.

34 Par commodité, nous avons remplacé le système de sigles de Héron (lettres grecques employées par 2 ou 4) par un système qui nous est propre.

${ }_{35}$ De ingeneis, Cod. lat. Monacensis $197 \Pi$ (I (BSBM), $\mathrm{f}^{\circ} 88 \mathrm{r}^{\circ}$.

36 De machinis, Ms.lat. 7239 (BNP), $\mathrm{f}^{\circ} 42 \mathrm{v}^{\circ}$.

\section{BIBLIOGRAPHIE}

Argoud, G. et J. Y. Guillaumin (1997) : Les pneumatiques d'Héron d'Alexandrie, texte et trad., Saint-Éticnne.

Beaujeu, J. (1950) : Pline. Histoire naturelle. Livre II, texte et trad., Paris.

Callebat, L. et Ph. Fleury (1986): Vitruve. De architectura. Livre X, texte, traduction et commentaires, CUF, Paris.

Ernout, A. et A. Meillet (1967) : Dictionnaire étymologique de la langue latine, Paris.

Forcellini, E. (1864) : Lexicon totius latinitatis, Padoue.

Martha, J. (1928) : Cicéron. De finibus, texte et traduction, CUF, Paris.

Oleson, J. P. (1984) : Greek and Roman Mechanical Water Liffing Devices : the History of Technology, Toronto

Oltramare, P. (1929) : Sénèque. Questions naturelles; Livre II, texte et traduction, CUF, Paris.

Rouanet, G. (1974): "Étude de quatre pompes à eau romaines provenant de l'épave Dramont D", CahArchSub, 3, 49-79.

Sablayrolles, P., avec la collaboration de M. Lacour (1988): "La pompe romaine de Périgueux", Aquitania, 6, 141-196.

Saint-Denis, E. de (1966) : Pline. Histoire naturelle. Livre XXXII, texte et traduction, CUF, Paris

Terquem, A. (1885) : La science romaine à l'époque d'Auguste. Étude historique d'après Vitruve, Paris. 